\title{
Scopolamine affects the cognitive processes involved in selective object exploration more than locomotor activity
}

\author{
M.-C. BUHOT \\ Laboratoire de Neurosciences Fonctionelles, C.N.R.S., Marseille, France \\ M. SOFFIE \\ Université Catholique de Louvain, Louvain-la-Neuve, Belgium \\ and \\ B. POUCET \\ Laboratoire de Neurosciences Fonctionelles, C.N.R.S., Marseille, France
}

\begin{abstract}
Three experiments were conducted to investigate the effects of a scopolamine injection $(1 \mathrm{mg} / \mathrm{kg}$, i.p.) on object exploration, response to change, and locomotor activity. Rats injected before exploration displayed a lower level of object investigation and a higher level of locomotor activity than did saline-injected controls (Experiment 1). Locomotor activity, but not object investigation, decreased with time. In addition, scopolamine-injected rats did not renew exploration after object changes. When treated after exploration (Experiment 2), scopolamine-injected rats showed a marked increase in object investigation, but not in locomotor activity. In this condition, they were able to detect a new object on the basis of its spatial location. The same pattern of overall activity was observed in Experiment 3 (a control condition with no change and animals treated after exploration). Taken together, these results suggest that the cholinergic system is more involved in cognitive components of exploration, such as the processing of object quality and location, than in sensorimotor processing. It is suggested that the memory deficits usually associated with an anticholinergic blockade might result from an impairment in the gathering and encoding of relevant information.
\end{abstract}

In recent years, the role of the cholinergic system in cognitive processes has been the focus of a great number of studies. This interest has mainly arisen from the observation that various human pathologies (e.g., Alzheimer's disease) are related to the selective dysfunctioning of the cholinergic system (Bartus, Dean, \& Flicker, 1987; Beatty, Butters, \& Janowsky, 1986; Davies \& Maloney, 1976), although a role for other neurotransmitters has also been suggested (Bowen et al., 1983; Vanderwolf, 1987; Wenk et al., 1987). In humans as well as in animals, cholinergic blockade impairs performance in a wide variety of learning and memory tasks (e.g., see Beatty \& Bierley, 1985; Burešová, Bolhuis, \& Bureš, 1986; Smith, 1988). In animals, these deficits are particularly evident in spatial situations. For example, anticho-

Funding for this project was provided by the Centre National de la Recherche Scientifique (C.N.R.S.). Part of this data was presented in a condensed form at the 2nd International Meeting of the European Behavioural Pharmacology Society (Athens, Greece, September 1988) and at the 18th Annual Meeting of the Society for Neuroscience (Toronto, November 1988). The authors wish to thank $M$. Renner and anonymous reviewers for their useful comments on the manuscript. All correspondence may be sent to M.-C. Buhot, Laboratoire de Neurosciences Fonctionelles, C.N.R.S., LNF U1bis, 31 Chemin Joseph-Aiguier, 13402 Marseille, France. linergic treatments have been found to markedly impair rats' performance in spatial navigation (Whishaw, 1985; Willner, Wise, \& Ellis, 1986), maze learning (Spangler, Rigby, \& Ingram, 1986), as well as in other spatial memory tasks (Beatty \& Bierley, 1986; Ellen, Taylor, \& Wages, 1986; Lerer, Warber, Friedman, Vincent, \& Gamsu, 1985; Lukaszewska \& Dlawichowska, 1985). From these results, however, it cannot be determined whether these impairments are due to (1) a specific inability to solve spatial problems (Ellen et al., 1986; Whishaw, 1985), (2) a general inability to perform complex tasks (Soffié, Bronchart, \& Lebailly, 1986), or (3) a selective impairment of spatial "working-memory" processes (Beatty \& Bierley, 1985).

A possible means for addressing this issue might rely on the use of new spatial and nonspatial tasks. One common characteristic of most previous studies aimed at assessing anticholinergic effects on cognitive processes is that they employed reinforcement contingencies that affect the animal's motivational state and, consequently, may restrict the animal's degree of freedom. For example, fear (e.g., of electric shocks), hunger, and thirst may greatly interfere with the animal's capacity to pay attention to a multiplicity of environmental features. Since attentional processes and motivational states are almost cer- 
tainly related, it can be argued that using a less constrained task could provide useful additional information relevant to the questions raised above.

Accordingly, in the present study, we assessed the effects of a systemic anticholinergic treatment using a newly developed task that minimizes the manipulation of the animal's motivation. Exploratory behavior is a spontaneous activity during which the animal learns about its environment. Several studies have previously documented the fact that animals given an exploratory experience with an environment will be more likely to display adaptive behavior (e.g., in escape or in finding food) than will naive animals (Ellen, Parko, Wages, Doherty, \& Herrmann, 1982; Renner, 1988).

When placed in a new environment, most mammals display marked exploratory reactions oriented toward some aspects of this environment. This activity decreases over time (habituation). However, if a change occurs (e.g., introduction of a new object or displacement of a familiar one), a renewal of exploration is usually observed. Moreover, this reinduced exploratory activity is mainly oriented toward the change (Poucet, Chapuis, Durup, \& Thinus-Blanc, 1986; Thinus-Blanc et al., 1987). Such renewal implies that some internal representation of the object configuration has been built up (perhaps in the form of a "cognitive map") and compared with the new arrangement. In the present study, two situations were contrasted. In the first situation (spatial change), the animals had to detect the change solely on the basis of spatial information, whereas in the second situation (object change), they could use both the location and the quality of the new object.

It is well documented that scopolamine, a muscarinic cholinergic antagonist, markedly increases locomotor activity in rats (e.g., see Sanberg, Henault, HagenmeyerHauser, \& Russell, 1987) and reduces habituation (Carlton, 1968). Accordingly, one concern of the present study was to dissociate scopolamine-induced changes in locomotor activity from more "cognitive" activities involved in object investigation.

\section{GENERAL METHOD}

\section{Subjects}

Across the three experiments, 58 naive male Long-Evans rats, about 90 days old and weighing $270-320 \mathrm{~g}$, were used. They were obtained from a laboratory-animal supplier (Janvier, St. Berthevin, France). All animals were housed in rectangular Plexiglas cages $(40 \times 30 \times 20 \mathrm{~cm}$ in height) in a colony room with natural lighting. They received food and water ad lib. Prior to testing, the anirmals were handled $10 \mathrm{~min}$ per day for 10 days and were randomly: assigned to either a control (saline-injected) or an experimental (scopolamine-injected) group.

\section{Apparatus}

The apparatus (shown in Figure 1) was a circular open field ( $160 \mathrm{~cm}$ in diameter with $30-\mathrm{cm}$-high walls) made of sheet metal. The open field was painted flat black and was surrounded by white curtains so that the environment was visually homogeneous except for a conspieuous striped pattern placed as shown in Figure 1. The objects that were placed in the open field were: (1) a Rubik's cube

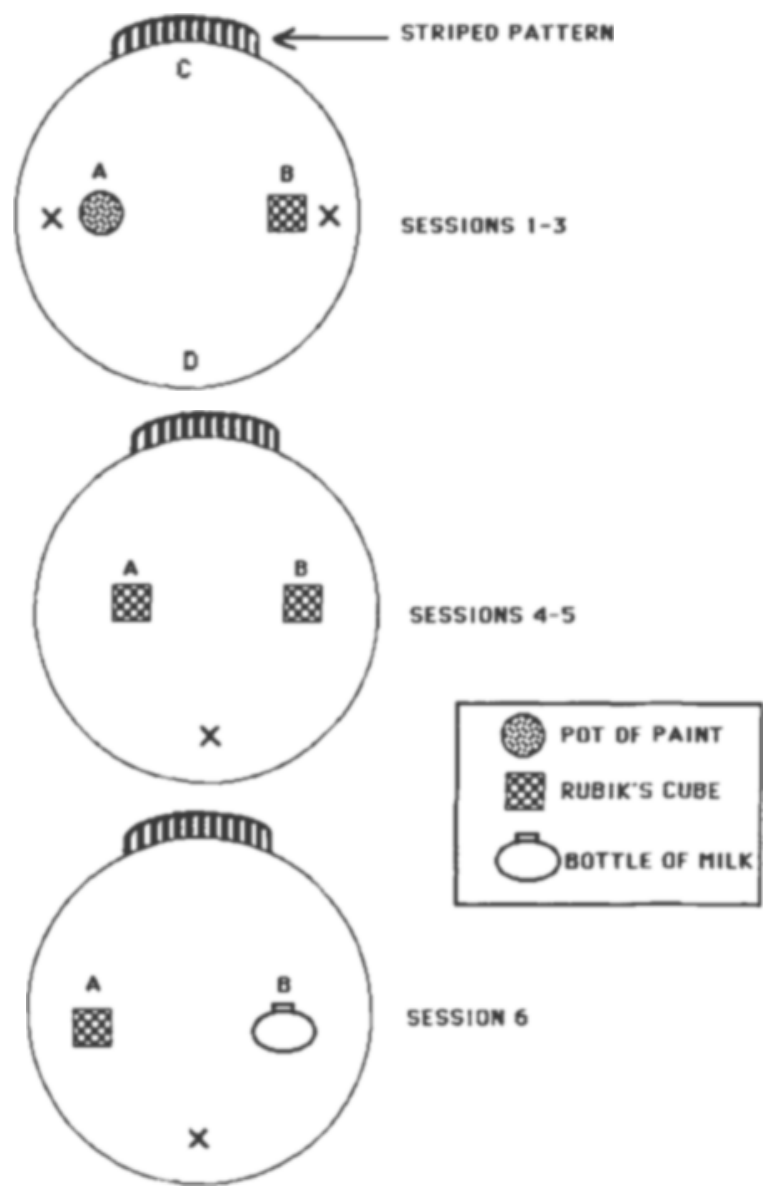

Figure 1. Schematic representation of the object configuration over successive sessions. The $\mathbf{X}$ symbol indicates the possible start locations. The probe locations under the open field are represented by $A, B, C$, and $D$ in the upper panel.

( $5.5 \mathrm{~cm}$ on each side) placed upon a cylindrical wooden base $(7.5 \mathrm{~cm}$ in diameter, $15 \mathrm{~cm}$ in height); (2) a cylindrical, fancy-colored pot of paint $(6.5 \mathrm{~cm}$ in diameter, $20 \mathrm{~cm}$ in height); or (3) a white, opaque bottle of milk $(8 \mathrm{~cm}$ in diameter, $20 \mathrm{~cm}$ in height). When they were in the arena, the objects could occupy either Location A or Location B (see Figure 1). The distance between these two locations was $80 \mathrm{~cm}$, so that each object was equally distant from the periphery and from the center of the arena. The field was illuminated by four $60-\mathrm{W}$ bulbs, which provided indirect lighting of the arena (about $40 \mathrm{lux}$ ).

\section{Tracking Device}

Each animal carried a 1-mm-long radioactive wire source (1921ridium, obtained from Oris, France) emitting gamma rays of approximately 4-8 $\mu \mathrm{c}(148-296 \mathrm{kBq})$. This source was implanted subcutaneously under mild ether anaesthesia, in the skin of the throat in a median position, 1 week before the experiment. This radioelement did not compromise the animal's health and allowed it to move freely. Four scintillator probes placed vertically under the open field could detect the radioactive source of an animal, hence its presence in these places. These probes were located either directly under the objects (A and B in Figure 1) or in selected places at the periphery of the open field (C and D in Figure 1). The scintillators were connected to a microcomputer, which stored and processed the data (see Buhot, Rage, \& Segu, in press; Rage \& Renucci, 1985, for more details). The data obtained with this method allowed for locat- 
ing the animal's presence near an object. The animal's behavior was also recorded by a video camera (placed $1.80 \mathrm{~m}$ above the arena) to allow verification of the data obtained with the radiotracking system. The measures taken with the video system were the number and duration of contacts the animals made with the objects. Good agreement was found between the measures obtained with the two systems. The data obtained with the radio-tracking system, which indicated the time spent in proximity to some object, were highly correlated with the data of the video system and, therefore, were considered as reflecting a measure of attention being paid to some object (i.e., object exploration).

\section{Procedure}

Each animal was subjected to the following phases. One day before the experiment, 2 rats were placed together into the open field without any object for two 15 -min sessions, with a 3-min intersession interval. This constituted the familiarization phase. The experimental sessions were conducted on the morning of the following day. Each animal was individually placed into the open field for six successive 9 -min sessions, separated by 3 -min intervals. In Sessions 1-3, the pot of paint and the Rubik's cube were located in Locations A and B, respectively (see Figure 1). Note that, in fact, two cubes instead of one were used, each on alternating sessions, so that both had been explored at least once by the animals. In Experiments 1 and 2, the pot of paint was replaced by the second cube on Session 4 so that at that time, the open field contained two identical objects, one of which occupied a "familiar" place (Place B) since it had been there during the habituation sessions (Sessions 1-3), whereas the other was in an "unfamiliar' place (Place A). Since an animal had to take the location of the changed object into account in order to detect the change, this first test constituted a "spatial" change. Session 5 was merely a replication of Session 4 (i.e., the two cubes remained in their places). On Session 6 , a completely new object (the bottle of milk) replaced the Rubik's cube in Location B (object change). In Experiment 3, the initial arrangement shown in Figure 1 (upper panel) remained unchanged throughout the six sessions. In many aspects, this procedure was logically similar to the response-to-change procedure in a T maze (Lukaszewska \& Dlawichowska, 1985).

During the habituation sessions (Sessions 1-3), half of the animals were introduced into the apparatus near Place A, the other half near Place B. In contrast, during the following sessions (Sessions 4-6), all animals were introduced into the apparatus at an intermediary location (see Figure 1, middle and bottom panels). This procedure was adopted in order not to bias the subjects toward exploring any particular object. During each 3-min interval between two consecutive sessions, the rats were placed into a black, cloth-covered waiting cage. The apparatus was cleaned between subjects but not between sessions for a given subject.

\section{Data Collection and Analysis}

A microprocessor recorded the durations of successive approaches to the different objects or to the two previously defined empty locations, $C$ and $D$. The variable retained for behavioral analyses was the time spent by an animal in close proximity to an object. Therefore, sampling measures were made before the experiment for each animal in order to determine the percentage of radioactivity detected by the probes when such behavior occurred. These measures were performed after the familiarization phase on the day preceding the experiment, and consisted of placing the animal in a small box with a $4 \times 4 \mathrm{~cm}$ opening for the animal's head. Measurements of radioactivity were taken when the animal's nose was in contact with the object. This procedure lasted for about $5 \mathrm{~min}$ and was not found to result in stressing the animals. These data were transformed into a percentage of radioactivity, which was used as a criterion of ob- ject investigation. For subsequent analyses, only values higher than this criterion were taken into account. In practice, these values corresponded to a concentric area around the object. As the rat was leaving this area, the percentage of radioactivity decreased in an exponential manner. For example, if the percentage of radioactivity was $80 \%$ when the animal's throat was $2 \mathrm{~cm}$ from an object, it decreased to $20 \%$ or to $2 \%$ when the subject was 4 or $8 \mathrm{~cm}$ from the object, respectively. Data from Locations $C$ and $D$ provided an index of locomotor activity at the periphery of the open field, which was calculated by counting the number of transitions between both locations. In that manner, it was possible to dissociate diffuse locomotor activity (as measured by activity between two "neutral" locations, C and D, with no object) from exploratory activity oriented toward the objects located in Locations $A$ and $B$.

Repeated measures analyses of variance (using a VAR 3 program; Rouanet \& Lépine, 1970) were carried out on these data, with pharmacological treatment as the between-subjects measure and successive sessions as the within-subjects measure. The VAR 3 program provided detailed analyses of the significant effects. It also allowed for restricted between-group comparisons on any session, as well as within-group comparisons in order to determine the time course of exploration and locomotor activity within each group (see Thinus-Blanc et al, 1987, for more details). These restricted analyses were basically equivalent to Student's $t$ tests. Habituation was assessed by comparing data from Session 1 with data from Session 3. Behavior in response to change was usually analyzed by comparing exploration during Sessions 3 and 4 (first change), and by comparing exploration during Sessions 5 and 6 (second change). However, for the first change of Experiment 1, Sessions 2 and 3 were pooled and were compared with Sessions 4 and 5 (see below).

\section{Pharmacological Treatment}

Injections were performed either $15 \mathrm{~min}$ before Session 1 (Experiment 1) or just after the end of Session 3 (Experiments 2 and 3). Accordingly, in Experiments 2 and 3, the interval between Sessions 3 and 4 was $15 \mathrm{~min}$ instead of $3 \mathrm{~min}$. The experimental subjects were injected (i.p.) with $1 \mathrm{mg} / \mathrm{kg}$ of scopolamine hydrochloride (Sigma Chemical Co., St. Louis, MO) dissolved in $2.5 \mathrm{ml}$ of normal saline $(0.9 \% \mathrm{NaCl})$. The drug was prepared fresh daily. Control animals received an equivalent volume of saline. Each day, 1 control (Sal) and 1 experimental (Scopo) animal were tested. Since it is well established that scopolamine does not exert its effects on memory via its peripheral actions (Beatty \& Bierley, 1985), methylscopolamine control groups were not included in the present study.

\section{EXPERIMENT 1}

\section{Method}

In this experiment, rats (Scopo, $n=10 ; \mathrm{Sal}, n=10)$ were injected $15 \mathrm{~min}$ prior to Session 1 and then received six sessions of exploration. The experimental design was as in Figure 1.

\section{Results}

\section{Object Exploration}

Figure 2 (upper panel) shows the time spent by the animals investigating the objects over each successive session. The analysis of variance revealed a significant main effect only for session $[F(5,90)=3.18, p<.02]$. Scopo animals were found to explore significantly less than Sal animals in Session 1 only $[F(1,18)=7.45, p<.02]$. In addition, only Sal subjects displayed a significant decrease (habituation) of object exploration from Session 1 to Ses- 

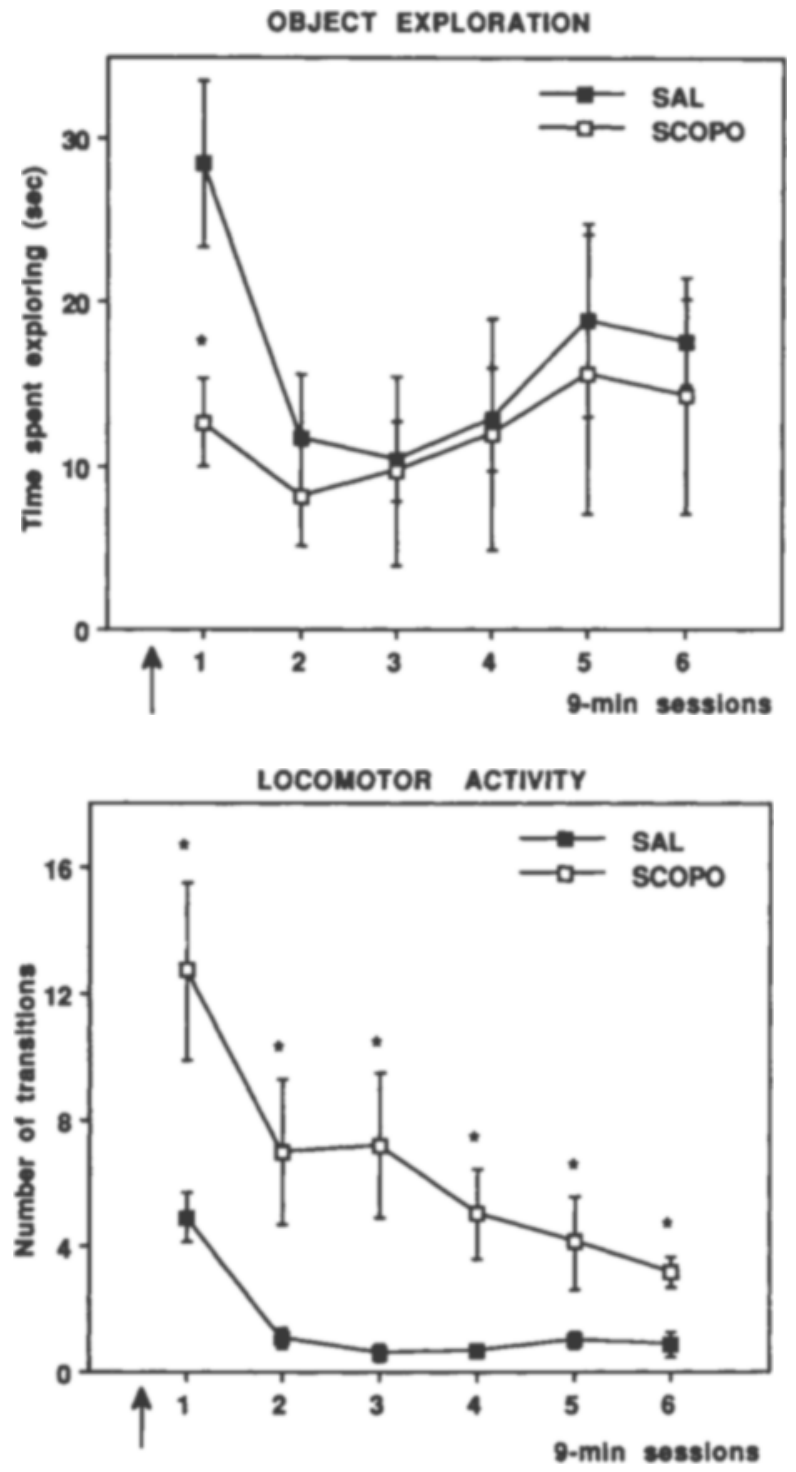

Figure 2. Object exploration (upper panel) and locomotor activity (number of transitions between Locations $C$ and D) at the periphery of the arena (lower panel) in Experiment 1. Significant differences between scopolamine-treated (Scopo; open squares) and salinetreated (Sal; filled squares) animals are indicated by * $(p<.05)$. The arrrow indicates the relative time of injection.

sion $3[F(1,9)=11.72, p<.01]$. Scopo animals, in contrast, did not habituate $(F<1)$.

\section{Locomotor Activity}

Figure 2 (lower panel) displays the mean number of transitions between Locations $C$ and D over successive sessions. The analysis of variance revealed a significant main effect for group $[F(1,18)=12.66, p<.01]$ and session $[F(5,90)=10.06, p<.001]$, and significant between-group differences on each successive session (see Figure 2). Scopo animals were more active than Sal animals on each session. In addition, a significant effect of session was found in both Sal $[F(5,45)=16.25, p<$ $.001]$ and Scopo animals $[F(5,45)=5.19, p<.001]$, providing evidence that both groups decreased their activity over sessions.

\section{Behavior in Response to Change}

First (spatial) change. A preliminary comparison of exploration during Sessions 3 and 4 did not reveal any significant effect of the first change in either group. Post hoc analyses revealed that this lack of reaction resulted from the fact that (1) the reactions were selectively oriented toward the change (i.e., the object in the unfamiliar place), and (2) some animals were observed to display reactions during Session 4, whereas others displayed reactions during Session 5. Accordingly, we conducted separate analyses for the changed object and for the unchanged object. In addition, data from Sessions 4 and 5 were pooled and compared with the pooled data from Sessions 2 and 3. The differences between Sessions $4+5$ and Sessions $2+3$ in time spent investigating either the changed or the unchanged object are presented in Figure 3 (left-hand histograms). The analysis of variance revealed a significant renewal of exploration toward the changed object in Sal animals $[F(1,9)=5.91, p<.05]$, but no such effect in Scopo animals $(F<1)$. No renewal of exploration of the unchanged object was found in either group [Sal, $F<1$; Scopo, $F(1,9)=1.77$, n.s.].

Second (object) change. The right-hand histograms of Figure 3 show the renewal of exploration induced by the new object placed in Location B during Session 6. Again, the analysis revealed a significant increase of exploration of the new object in Sal animals $[F(1,9)=11.42, p<$ $.01]$, but no such effect in Scopo animals $(F<1)$. No significant effect of the unchanged object was found in either group [Sal, $F(1,9)=2.23$, n.s.; Scopo, $F(1,9)=$ 1.83 , n.s.].

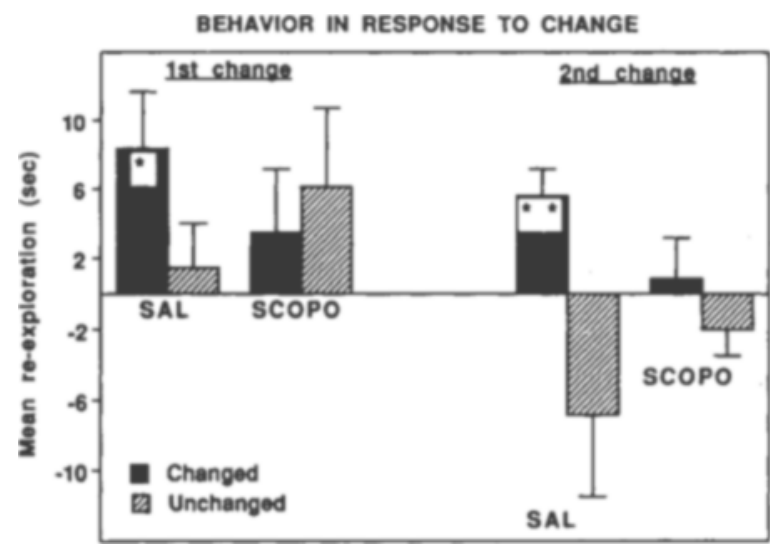

Figure 3. Behavior in response to change in Experiment 1. The histograms represent the differences in exploratory activity between postchange session(s) and prechange session(s). A positive score means renewed exploration on the change session(s). Left panel: first ("spatial") change (Sessions 4 and 5 vs. Sessions 2 and 3). Right panel: second ("object") change (Session 6 vs. Session 5). Significant variations in exploration are indicated by $*(p<.05)$ and ** $(p<.01)$. 


\section{Discussion}

Compared with saline-injected animals, scopolaminetreated rats displayed a lower level of initial object investigation and did not habituate. Furthermore, they did not react to a familiar object in an unfamiliar location (first change), nor did they react to a completely new object (second change). Therefore, the effects of central cholinergic blockade might not be specific to the spatial domain, but seem to affect more general informationprocessing systems. In contrast, locomotor activity was markedly increased in treated animals relative to controls. However, locomotor activity in the treated rats decreased over sessions. Overall, this pattern of results suggests that the scopolamine treatment disturbed the processing of object quality and location. In Experiment 2, we asked whether a posthabituation treatment, which does not affect initial encoding, would produce similar effects on behavior in response to change.

\section{EXPERIMENT 2}

\section{Method}

Rats (Scopo, $n=10$; Sal, $n=10$ ) were injected after habituation at the end of Session 3. Session 4 (change session) occurred $15 \mathrm{~min}$ after the injection. Except for this particular intersession interval, the animals were subjected to six 9-min sessions separated by 3-min intervals, following the same design as in Experiment 1 (see Figure 1).

\section{Results}

\section{Object Exploration}

Figure 4 (upper panel) presents the time course of exploration over successive sessions. No significant effects were found for the main factor of either session $[F(5,90)$ $=1.56$, n.s. $]$ or group $[F(1,18)=1.41$, n.s. $]$. Nevertheless, a significant session $\times$ group interaction was found $[F(5,90)=4.09, p<.01]$. Additional analyses revealed no differences between the Sal and Scopo animals in Sessions 1-3 (during which the animals were untreated). All subjects decreased their object exploration from Session 1 to Session 3 [Sal, $F(1,9)=5.23, p<.05$; Scopo, $F(1,9)=8.27, p<.02]$. Although the Scopo animals displayed a marked increase in object exploration following the injection (upper panel of Figure 4), this increase was not statistically significant [Session 3 vs. Session 4 , $F(1,9)=2.75$, n.s.], due to a high level of individual variation. This enhanced activity did not decrease on subsequent sessions, and in Session 6, Scopo animals were found to be significantly more active than Sal subjects $[F(1,18)=5.13, p<.05]$.

\section{Locomotor Activity}

Measurements of locomotor activity over successive sessions are shown in Figure 4 (lower panel). The analysis of variance revealed a significant main effect for session $[F(5,90)=39.61, p<.001]$, but no effect for group $(F<1)$ and no significant group $\times$ session interaction $[F(5,90)=1.12$, n.s. $]$. A significant effect of session was found in both $\mathrm{Sal}[F(5,45)=47.29, p<.001]$ and Scopo
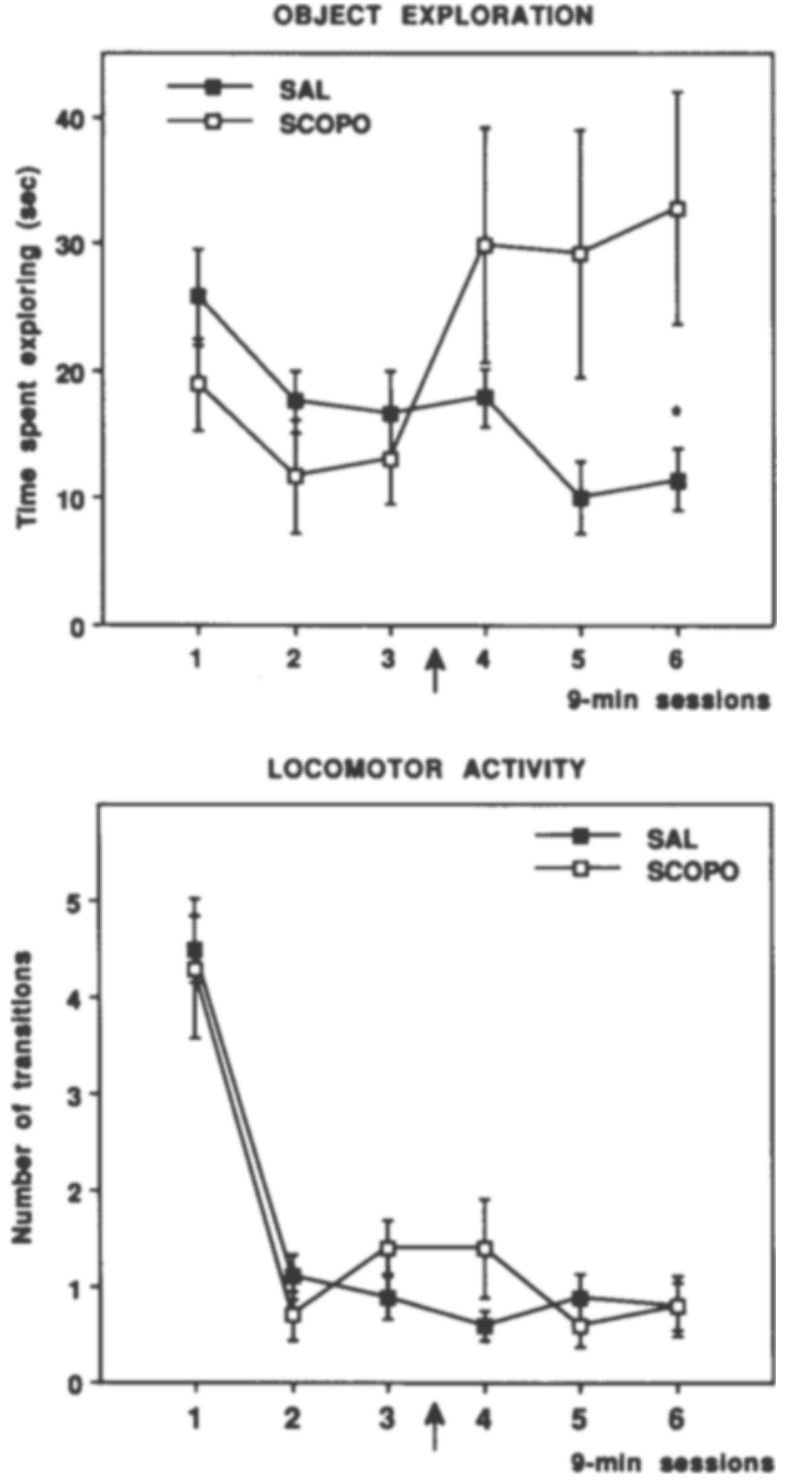

Figure 4. Object exploration (upper panel) and locomotor activity (number of transitions between Locations $C$ and D) at the periphery of the arens (lower panel) in Experiment 2. Significant differences between scopolamine-treated (Scopo; open squares) and salinetreated (Sal; filled squares) animals are indicated by $*(p<.05)$. The arrow indicates the relative time of injection.

animals $[F(5,45)=12.34, p<.001]$; both groups decreased their activity over sessions.

\section{Behavior in Response to Change}

First (spatial) change. The differences between Session 4 (after the change) and Session 3 (before the change) in time spent investigating either the changed or the unchanged object are presented in Figure 5 (left-hand histograms). The comparison between Sessions 3 and 4 revealed a significant renewal of exploration toward the changed object both in $\mathrm{Sal}[F(1,9)=6.84, p<.05]$ and Scopo animals $[F(1,9)=5.16, p<.05]$. No significant 
BEHAVIOR IN RESPONSE TO CHANGE

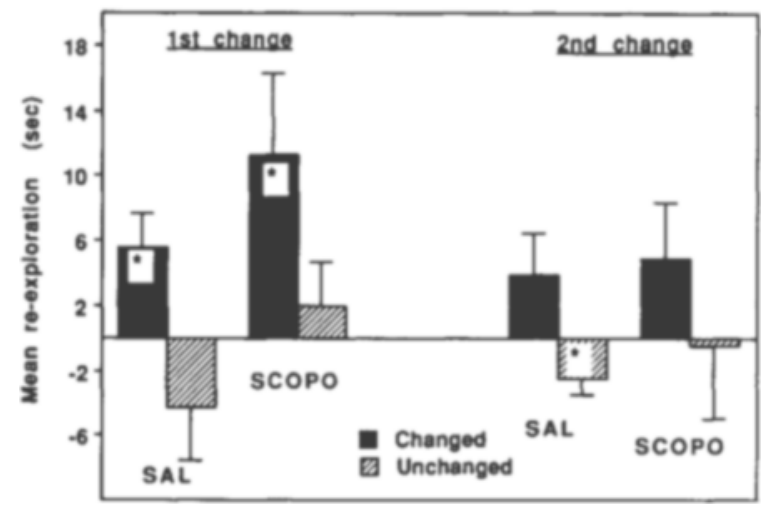

Figure 5. Behavior in response to change in Experiment 2. The histograms represent the differences in exploratory activity between the postchange session and the prechange session. A positive score means renewed exploration on the change sessions. Left panel: first ("spatial") change (Session 4 vs. Session 3). Right panel: second ("object") change (Session 6 vs. Session 5). Significant variations in exploration are indicated by $*(p<.05)$.

remewal of exploration of the unchanged object was found in either group [Sal, $F(1,9)=1.61$, n.s.; Scopo, $F<1]$.

Second (object) change. Figure 5 (right-hand histograms) shows the change-induced exploratory activity during Session 6. No significant increase in exploration of the new object was found in either group [Sal, $F(1,9)=$ 2.43 , n.s.; Scopo, $F(1,9)=1.51$, n.s.]. Despite this apparent lack of reaction to the change, the Sal animals were observed to spend more time exploring the new object $(M=9.15 \mathrm{sec})$ than the familiar one $(M=2.35 \mathrm{sec})$ during Session $6[F(1,9)=15.52, p<.01]$, which was not the case in Session 5 (Object A, $M=5.2 \mathrm{sec}$; Object B, $M=4.85 \mathrm{sec} ; F<1)$. No preference for the changed object was found in the Scopo animals during Session 6 (changed object, $M=17.5 \mathrm{sec}$; unchanged object, $M=$ $16.2 \mathrm{sec} ; F<1$ ). In addition, the unchanged object was investigated less during Session 6 than during Session 5 by Sal animals only $[F(1,9)=6.82, p<.05]$.

\section{Discussion}

In contrast to Experiment 1, the anticholinergic treatment was found to result in a normal pattern of locomotor activity and in increased, rather than decreased, objectoriented exploratory activity. To some extent, the latter result is consistent with the observation made by Horsburgh and Hughes (1981) that scopolamine induces novelty avoidance and familiarity preference. However, this explanation in terms of novelty/familiarity is somewhat contradicted by the selective reexploration of the changed object (revealing a novelty preference) in Scopo animals during Session 4 . Moreover, this selective reexploration suggests that treated animals are still able to use relevant information gathered prior to drug injection. A similar explanation accounts for the Scopo animals' lack of reaction to the object change in Session 6, since they were drugged when they had to gather the relevant information (Sessions 4-5). The fact that the Scopo animals did not decrease their exploratory activity from Sessions 4 to 6 gives some credence to this hypothesis, insofar as such a result indicates an impairment in habituation, and therefore in information integration and processing (O'Keefe \& Nadel, 1978). It is interesting to note that, although the saline-control animals in Experiment 1 distributed their renewal of exploration in both Sessions 4 and 5 , in Experiment 2 they reacted immediately to the spatial change during Session 4 . This result could be due either to a nonspecific injection effect that might have increased their level of arousal in Session 4, or to an effect of the between-session interval ( $15 \mathrm{vs} .3 \mathrm{~min})$, which might have favored consolidation of memory.

In summary, Experiments 1 and 2 produced contrasting results, as indicated by the interaction between drug effects and relative time of injection on the amount of exploration. Experiment 3 was undertaken to further document this interaction. In particular, since the increased amount of exploration observed in Experiment 2 after a scopolamine injection could have resulted either from the change or from the treatment itself (both factors were confounded in the design of Experiment 2), it was not possible to dissociate the respective influence of each factor. Accordingly, in Experiment 3, all animals were tested under a condition in which no change occurred at any time.

\section{EXPERIMENT 3}

\section{Method}

Experiment 3 was a replication of Experiment 2, with the exception that no change was made to the initial situation in any session. Thus, the configuration shown in the upper panel of Figure 1 was used throughout the six sessions. Rats (Scopo, $n=9$; Sal, $n$ $=9$ ) were injected after Session 3, with Session 4 occurring $15 \mathrm{~min}$ later.

\section{Results}

\section{Object Exploration}

Figure 6 (upper panel) displays the time course of exploratory activity over successive sessions. The analysis of variance performed on this data showed significant effects for group $[F(1,16)=10.29, p<.01]$ and session $[F(5,80)=3.44, p<.01]$, and a significant group $\times$ session interaction $[F(5,80)=10.14, p<.001]$. Since no significant differences between the two groups were observed from Sessions 1 to 3 , this significant interaction can be accounted for by a marked increase in exploratory activity by the Scopo animals in Session 4 , which followed the injection $[F(1,8)=28.42, p<.001]$. Note that a similar, but nonsignificant, increase was also observed in the Sal animals $[F(1,8)=1.65$, n.s.]. However, although this increase was temporary and was followed by a subsequent decrease in the Sal animals [Session 4 vs. Session $6, F(1,8)=9.07, p<.02$ ], enhanced activity by the Scopo animals lasted until the end of the experiment. In addition, there was no significant difference between Sessions 4 and $6[F(1,8)=1.87$, n.s. $]$. The 

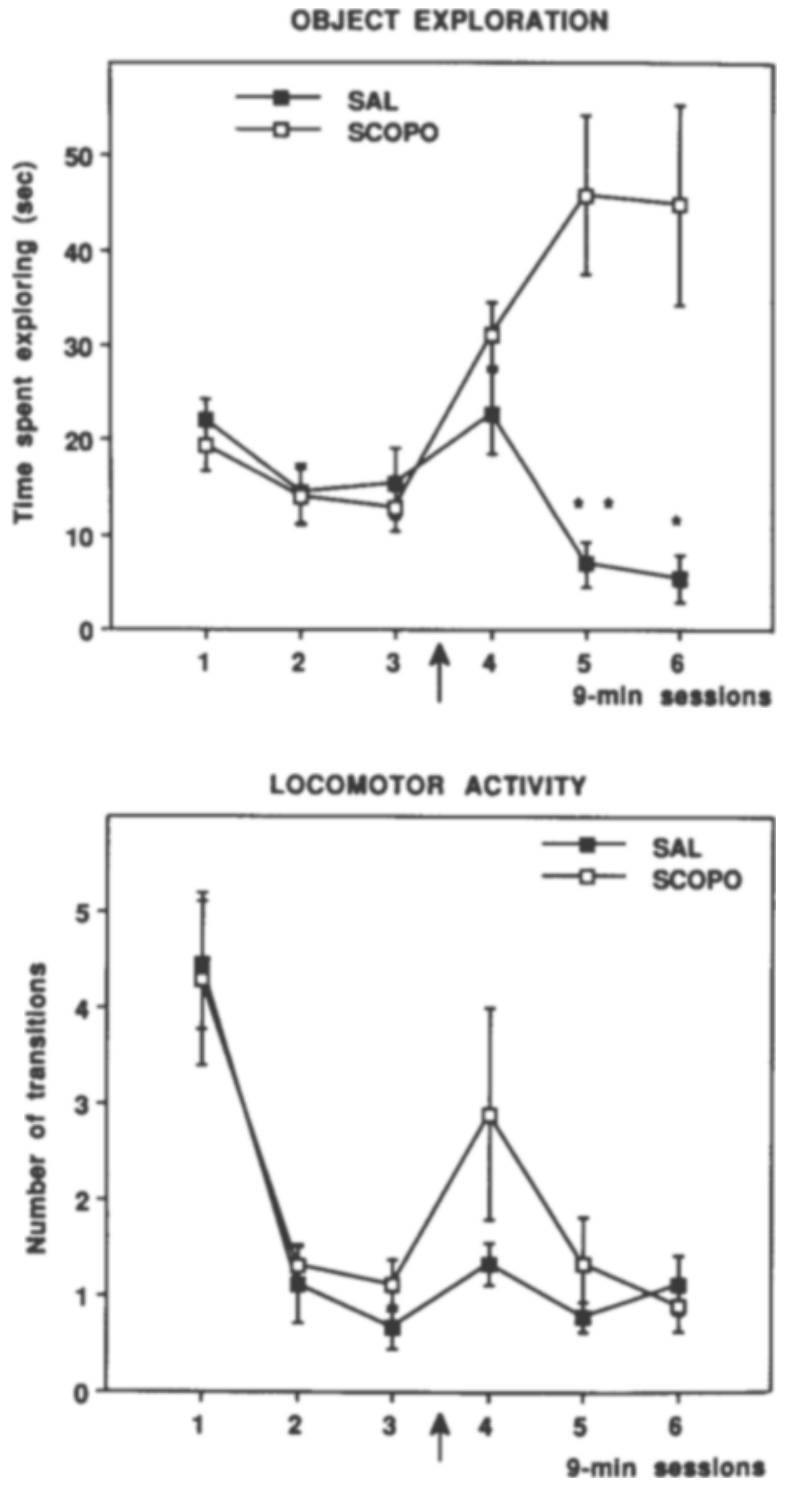

Figure 6. Object exploration (upper panel) and locomotor activity (number of transitions between Locations $C$ and D) at the periphery of the arena (lower panel) in Experiment 3. Significant differences between scopolamine-treated (Scopo; open squares) and salinetreated (Sal; filled squares) animals are indicated by $*(p<.01)$ and $* *(p<.001)$. The arrow indicates the relative time of injection.

Scopo animals were significantly more active than the Sal animals in Sessions $5[F(1,16)=19.38, p<.001]$ and $6[F(1,16)=13.12, p<.01]$.

Finally, in order to obtain control data for the changeinduced reactions of the Scopo animals in Experiment 2, similar analyses, which separately processed the two objects, were undertaken (although no change was made at any time in Experiment 3). Although the saline injection did not result in any significant increase in exploration of either object [Session 3 vs. Session 4: Object A, $F<1$; Object B, $F(1,8)=3.47$, n.s.], the scopolamine treatment was found to induce a significant renewal of exploration of each object [Session 3 vs. Session $4: \mathrm{Ob}-$ ject $\mathrm{A}, F(1,8)=13.40, p<.01$; Object $\mathrm{B}, F(1,8)=$ $6.94, p<.05]$. In contrast to Experiment 2, however, no indication was found that the renewal observed in Session 4 was selectively oriented to any particular object.

\section{Locomotor Activity}

The pattern of locomotor activity was quite similar to that observed in Experiment 2 (Figure 6, lower panel). A significant effect of session was found in both the Sal $[F(5,40)=13.56, p<.001]$ and the Scopo animals $[F(5,40)=5.40, p<.001]$.

\section{Discussion}

Even when no change occurred, the Scopo animals demonstrated a remarkable treatment-induced increase in object exploration. Moreover, the fact that Experiment 3 yielded a diffuse renewal of exploration, rather than a selective renewal as in Experiment 2, suggests that the change brought to the initial situation in Experiment 2 was actually detected by the Scopo animals. Thus, the increase obtained in Experiment 2 was not due solely to an effect of the injection. These results confirm that scopolamine does not alter novelty/familiarity judgments, and therefore they are difficult to account for by impaired memory (Horsburgh \& Hughes, 1981).

Additional analyses revealed a slight (nonsignificant) increase of exploration in the saline animals in Session 4, suggesting that a nonspecific injection effect on the level of arousal could account for the immediate renewal of exploration in Experiment 2 as opposed to the delayed renewal in Experiment 1. However, a possible role of the between-session interval still cannot be discarded.

\section{GENERAL DISCUSSION}

This study clearly emphasizes the validity of our experimental design for assessing behavioral changes following pharmacological treatments (see also Buhot et al., in press), since it allows dissociation of locomotor activity from exploration (Leyland, Robbins, \& Iversen, 1976). More specifically, if habituation reflects the integration of information (O'Keefe \& Nadel, 1978), the time course of object exploration could constitute an appropriate model of memory. However, only subsequent tests of response to change can reveal whether, indeed, such processing has occurred. We have previously demonstrated that, by manipulating the nature of the changed information, it is further possible to determine the characteristics of the environment that are actually processed by an animal (e.g., see Thinus-Blanc et al., 1987).

On the basis of these observations, this study revealed that scopolamine (1) differentially affects locomotor activity and the cognitive components of the task relating to object investigation, and (2) acts differently depending on when it is injected. In all three experiments, scopolamine was found to enhance exploratory activity. However, when the drug was injected prior to initial 
familiarization, this enhancement concerned the nonspecific aspects of the environment, resulting in an increase in locomotor activity. In contrast, when injected after habituation, this enhancement concerned investigation of the objects. Thus, cholinergic blockade appears to disinhibit exploratory responses, although this disinhibition is expressed in different ways according to the degree of the animal's familiarity with the environment. This conclusion is reminiscent of Gray's (1984) hypothesis that the cholinergic septohippocampal pathway would control a behavioral inhibition system aimed at interrupting ongoing behaviors when an animal confronts unexpected or aversive stimuli. In our experiments, it can be hypothesized that the scopolamine-induced dysfunctioning of the septohippocampal system induces a disinhibition of normal exploratory behavior, and thus results in an exaggerated and imbalanced pattern of exploration. In this respect, it is interesting to note that the pattern of exploration presented by scopolamine-injected rats resembles much more that of septum-damaged rats than that of medial frontal cortex-damaged rats (Poucet, 1989; Poucet, Thinus-Blanc, \& Buhot, 1988). This result gives some additional support to the hypothesis that the cholinergic septohippocampal pathway is crucially involved in the control of processes that regulate exploration and habituation.

Although this interpretation in terms of disinhibition can account for the observed interaction between the drug effect on the qualitative/quantitative aspects of exploration and the degree of the animal's familiarity with the environment, it cannot explain (1) the presence of a habituation pattern of locomotor activity, but not of object-oriented activity, following the scopolamine injection in all experiments; (2) the failure of prehabituation-treated rats to react to both spatial and object changes (Experiment 1); and (3) the apparent contradiction between the scopolamineinjected rats' ability to react to the spatial change and their inability to react to the object change, when treated after habituation (Experiment 2).

To account for these results, it appears necessary to postulate that scopolamine also produces cognitive deficits, in addition to its disinhibitory effects. In particular, Experiment 2 revealed a selective renewal of exploration toward the changed object by scopolamine-injected animals in Session 4, a result which suggests that, if an animal has had the opportunity to integrate relevant information when not under the influence of the drug, it is still able to use that information despite cholinergic blockade. Conversely, the treated rats' failure to react to the object change in Session 6 of Experiment 2 probably resulted from the fact that they were injected at the encoding stages (i.e., Sessions 4-5). Therefore, if the cholinergic system plays a role in cognitive functions, this role would mainly concern the initial encoding of information, rather than the storage and/or retrieval of information. This conclusion is further supported by the failure of the treated animals to habituate their object explora- tion. It should be noted that such deficits in habituation cannot be easily explained by floor effects due to lower activity levels in scopolamine-injected animals, since even when they were very active, no habituation was found (Experiments 2 and 3, Sessions 4-6). The encoding hypothesis is also supported by both animal (Soffié, 1988; Soffié et al., 1986) and human (Ghoneim \& Mewaldt, $1975,1977)$ studies showing that scopolamine produces deficits when injected before initial acquisition, but not when injected between acquisition and retention. Although some authors have also argued for a role of the cholinergic system in retrieval (e.g., Beatty \& Bierley, 1986), such a retrieval function is not supported by the observation that the treated animals reacted to the spatial change in Experiment 2 . Finally, a possible role of the cholinergic system in storage and consolidation appears to be ruled out by the present and other studies (Beatty \& Bierley, 1986; Ridley, Bowes, Baker, \& Crow, 1984).

Several authors have also pointed out a possible role of the cholinergic system in attentional processes (Warburton, 1977) or in maintenance of attention (Cheal, 1981). Although our results are consistent with such hypotheses, they suggest that the scopolamine-induced deficits would rely on an imbalance of attention, rather than on its maintenance. As discussed above, such an imbalance of attention would result from the scopolomineinduced disinhibition. Inhibition is necessarily involved in the regulation of exploration of a novel environment (llyutchenok, 1973), since it allows the animal to select (and therefore encode) the relevant information. Thus, the present data are consistent with a hypothesis of cholinergic involvement in gathering and encoding relevant information, rather than in memory processes per se. The cholinergic system would play a major role in attentional processes by exerting an inhibitory influence on the response systems, which are triggered by highly emotional states.

\section{REFERENCES}

Bartus, R. T., Dean, R. L., \& Flicker, C. (1987). Cholinergic pharmacology: An integration of human and animal research on memory. In H. Y. Meltzer (Ed.), Psychopharmacology: The third generation of progress (pp. 219-231). New York: Raven.

BEATTY, W. W., \& BiERLEY, R. A. (1985). Scopolamine degrades spatial working memory but spares spatial reference memory: Dissimilarity of anticholinergic effects and restriction of distal visual cues. Pharmacology, Biochemistry, \& Behavior, 23, 1-6.

Beatty, W. W., Bierley, R. A. (1986). Scopolamine impairs encoding and retrieval of spatial working memory in rats. Physiological Psychology, 14, 82-86.

Beatty, W. W., Butters, N., \&anowsky, D. (1986). Patterns of memory failure after scopolamine treatment: Implications for cholinergic hypothesis of dementia. Behavioral \& Neural Biology, 45, 196-211.

Bowen, D. M., Allen, S. J., Benton, J. S., Goodhardt, M. J., Haan, E. A., Palmer, A. M., Sims, N. R., Smith, C. C. T., Spillane, J. A., Esiri, M. M., Neary, D., Snowdon, J. S., Wilcock, G. K., \& Davison, A. N. (1983). Biochemical assessment of serotonergic and cholinergic dysfunction and cerebral atrophy in Alzheimer's disease. Journal of Neurochemistry, 41, 266-272.

Buhot, M.-C., Rage, P., \&Égu, L. (in press). Changes in explora- 
tory behaviour of hamsters following treatment with 8-Hydroxy-2(di-n-propylamino)tetralin (8-OH-DPAT). Behavioural Brain Research.

Burešoví, O., Bolhuis, J., Bureš, J. (1986). Differential effects of cholinergic blockade on performance of rats in the water tank navigation task and in a radial arm maze. Behavioral Neuroscience, 100, 476-482.

Carlton, P. L. (1968). Brain-acetylcholine and habituation. In P. B. Bradley \& M. Fink (Eds.), Progress in brain research (Vol. 28, pp. 48-60). New York: Elsevier.

ChEAL, M. L. (1981). Scopolamine disrupts maintenance of attention rather than memory processes. Behavioral \& Neural Biology, 33. 163-187.

DAvies, P., \& MALONEY, A. J. F. (1976). Selective loss of central cholinergic neurons in Alzheimer's disease. Lancet, 2, 1403.

Ellen, P., Parko, E. M., Wages, C., Doherty, D., Herrmann, T. (1982). Spatial problem solving by rats: Exploration and cognitive maps. Learning \& Motivation, 13, 81-94.

Ellen, P., TAYloR, H. S., \& Wages, C. (1986). Cholinergic blockade effects on spatial integration versus cue discrimination performance. Behavioral Neuroscience, 100, 720-728.

GhoneIM, M. M., MewaldT, S. P. (1975). Effects of diazepam and scopolamine on storage, retrieval and organizational processes in memory. Psychopharmacologia, 44, 257-262.

GHoneim, M. M., \& MEWALDT, S. P. (1977). The interaction of scopolamine, diazepam and physostigmine in human memory. Psychopharmacologia, 52, 1-6.

GraY, J. A. (1984). The hippocampus as an interface between cognition and emotion. In H. L. Roitblat, T. J. Bever, \& H. S. Terrace (Eds.), Animal cognition (pp. 607-626). Hillsdale, NJ: Erlbaum.

HoRsBURGH, R. J., \& Hughes, R. N. (1981). Modification of novelty preferences in rats by current and prior treatment with scopolamine and methylscopolamine. Psychopharmacology, 73, 388-390.

ILYUTCHENOK, R. Y. (1973). Cholinergic brain mechanisms and memory. In G. Adams (Ed.), Biology of memory (pp. 239-246). New York: Plenum.

Lerer, B., Warber, J., Friedman, E., Vincent, G., \& Gamsu, E. (1985). Cortical cholinergic impairment and behavioral deficits produced by kainic acid lesions of rat magnocellular basal forebrain. Behavioral Neuroscience, 99, 661-677.

Leyland, M., Robeins, T., \& Iversen, S. D. (1976). Locomotor activity and exploration: The use of traditional manipulators to dissociate these two behaviors in the rat. Animal Learning \& Behavior, 4, 261-265.

LUKASZEWSKA, I., DLAWICHOWSKa, E. (1985). Scopolamine impairs the response-to-change following observation of the environment but not after its exploration by the rat. Physiology \& Behavior, 34, 625-629.

O'KeEFE, J., \& NADEL, L. (1978). The hippocampus as a cognitive map. Oxford: Clarendon Press.

PouCET, B. (1989). Object exploration, habituation and response to a spatial change in rats following septal or medial frontal cortical damage. Behavioral Neuroscience, 103(5).

Poucet, B., Chapuis, N., Durup, M., \& Thinus-Blanc, C. (1986). A study of exploratory behavior as an index of spatial knowledge in hamsters. Animal Learning \& Behavior, 14, 93-100.
Poucet, B., Thinus-Blanc, C., \& Buhot, M.-C. (1988). Object exploration, habituation and response to change in rats following frontal cortex lesions, septal lesions, or scopolamine injections. Society for Neuroscience Abstracts, 14, 1229.

RAGe, P., RENucci, M. (1985). Saisie de données dans l'étude du comportement animal. Electronique Applications, 44, 87-92.

RENNER, M. J. (1988). Learning during exploration: The role of behavioral topography during exploration in determining subsequent adaptive behavior. International Joumal of Comparative Psychology, 2, 43-56.

Ridley, R. M., Bowes, P. M., BAker, H. F., CRow, T. J. (1984). An involvement of acetylcholine in object discrimination learning and memory in the marmoset. Neuropsychologia, 22, 253-263.

RouANET, H., \& LépINE, D. (1970). Comparisons between treatments in a repeated measurement design: ANOVA and multivariate methods. British Joumal of Mathematical \& Statistical Psychology, 23, 146-163.

Sanberg, P. R., Henault, M. A., Hagenmeyer-Hauser, S. H., \& RusseLL, K. H. (1987). The topography of amphetamine- and scopolamine-induced hyperactivity: Towards an activity print. Be havioral Neuroscience, 101, 131-133.

SMITH, G. (1988). Animal models of Alzheimer's disease: Experimental cholinergic denervation. Brain Research Reviews, 13, 103-118.

SOFFÍ, M. (1988). Approche psychobiologique du vieillissement cérébral chez le rat: Implications et modulations cholinergiques. Doctoral dissertation, Université Catholique de Louvain, Louvain, Belgium.

Soffí, M., Bronchart, M., \& Lebailly, B. (1986). Scopolamineinduced deficits in acquisition of a complex spatial learning. Physiology \& Behavior, 37, 79-84.

Spangler, E. L., Rigby, P., \& Ingram, D. (1986). Scopolamine impairs learning performance of rats in a 14-unit T maze. Pharmacology, Biochemistry, \& Behavior, 25, 673-679.

Thinus-Blanc, C., Bouzouba, L., Chaix, K., Chapuis, N., Durup, M., \& Poucet, B. (1987). A study of spatial parameters encoded during exploration in hamsters. Journal of Experimental Psychology: Animal Behavior Processes, 13, 418-427.

VANDERWOLF, C. H. (1987). Near-total loss of "leaming" and "memory" as a result of combined cholinergic and serotonergic blockade in the rat. Behavioural Brain Research, 23, 43-57.

WARBuRton, D. M. (1977). Stimulus selection and behavioral inhibition. In L. L. Iversen, S. D. Iversen, \& S. H. Syder (Eds.), Handbook of psychopharmacology (Vol. 8, pp. 385-431). New York: Plenum.

Wenk, G., Hughey, D., Boundy, V., Kim, A., Walker, L., \& OLTON, D. S. (1987). Neurotransmitters and memory: Role of cholinergic, serotonergic and noradrenergic systems. Behavioral Neuroscience, 101, 325-332.

Whishaw, I. Q. (1985). Cholinergic receptor blockade in the rat impairs locale but not taxon strategies for place navigation in a swimming pool. Behavioral Neuroscience, 99, 979-1005.

WILlNER, P., WISE, D., \& El.Ls, T. (1986). Specific disruption of spatial behavior in rats by central muscarinic receptor blockade. Psychopharmacology, 90, 229-235.

(Manuscript received February 13, 1989; revision accepted for publication August 1, 1989.) 